\title{
Peste des Petits Ruminants Vaccine (Nigerian Strain 75/1) Confers Protection for at Least 3 Years in Sheep and Goats
}

\author{
Aamer Bin Zahur1, Hamid Irshad1 ${ }^{*}$, Aman Ullah', Muhammad Afzal'2, Asma Latif', \\ Riasat Wasee Ullah'1, Umer Farooq ${ }^{1}$, Muhammad Humayoon Samo², Muhammad Jahangir', \\ Giancarlo Ferrari ${ }^{3}$, Manzoor Hussain ${ }^{2}$, M. Munir Ahmad ${ }^{4}$ \\ ${ }^{1}$ Animal Health Research Laboratories, Animal Sciences Institute, National Agricultural Research Centre, \\ Islamabad, Pakistan \\ ${ }^{2}$ FAO-UN Pakistan (GCP/PAK/127/USA) NARC, Park Road, Islamabad, Pakistan \\ ${ }^{3} \mathrm{AGAH}, \mathrm{FAO}(\mathrm{Hq})$, Rome, Italy \\ ${ }^{4}$ Divisional Diagnostic Laboratory, Livestock and Dairy Development Department, Multan, Pakistan \\ Email: ${ }^{*}$ hamidirshad@hotmail.com
}

Received July 2014

\section{Abstract}

The present study reports the duration of immunity and protective efficacy of Peste des Petits Ruminants (PPR) vaccine (Nigerian strain 75/1) in sheep and goats. A total of 105 sheep and goats were divided into three groups $A, B$ and $C$. Group A received normal recommended dose $(1.0 \mathrm{ml})$ of PPR vaccine, group $B$ received half dose $(0.5 \mathrm{ml})$ of PPR vaccine and group $C$ was kept as unvaccinated control group in contact with vaccinated animals. The post vaccination dynamics of antibodies against PPR virus was studied. It was found that significant antibody titres persisted for 3 years post vaccination in sheep and goats vaccinated with either full dose or half dose of PPR vaccine. The challenge protection studies were carried out in experimental animals at 24 and 36 month post vaccination. The vaccinates withstood challenge and were found completely resistant clinically and virologically to virulent PPR virus for 24 and 36 months post vaccination. The unvaccinated control animals developed typical clinical signs of PPR and the challenged virus was detected in ocular, nasal and oral secretions of these animals. This study demonstrated that a single immunization with PPR vaccine conferred solid protection in sheep and goats for 3 years.

\section{Keywords}

PPR Vaccine, Small Ruminants

\section{Introduction}

Peste des petits ruminant (PPR) is an acknowledged acute and highly contagious newly emerging/evolving disease of small ruminants. The disease is caused by a Morbilli virus of genus paramyxoviridae [1]. Similar to oth-

\footnotetext{
"Corresponding author.

How to cite this paper: Zahur, A.B., et al. (2014) Peste des Petits Ruminants Vaccine (Nigerian Strain 75/1) Confers Protection for at Least 3 Years in Sheep and Goats. Journal of Biosciences and Medicines, 2, 27-33.

http://dx.doi.org/10.4236/jbm.2014.26005
} 
er Morbilli viruses it is capable of destroying the whole immunologically naive host population by provoking epidemics and pandemics subsequently damaging economy, undermining food security and livelihood of the poor farmers [2].

PPR was confirmed for the first time in Pakistan in 1994 when samples collected during a suspected outbreak were sent to the Institute of Animal Health, Pirbright UK [3]. The disease has been a cause of heavy mortality among small ruminants in Pakistan since last decade [4]. Since then number of PPR outbreaks have been recorded and now PPR appears to be widespread and endemic in Pakistan [5]. Previously tissue culture rinderpest vaccine (TCRV) was used for control of PPR in Pakistan. However, with the declaration of freedom from rinderpest in 2007 its use in Pakistan was discontinued. Now a homologous PPR vaccine (Nigerian strain 75/1) appears to be the only remedy in this regard. However, very limited information is available about the duration of immunity and protective efficacy of this vaccine. Therefore, a study was designed to evaluate the duration of immunity and protective efficacy of the homologous PPR vaccine. The information thus generated by this study would help in devising appropriate vaccination strategy for control of PPR in Pakistan.

\section{Material and Methods}

\subsection{Experimental Animals}

Sheep $(n=45)$ and goats $(n=60)$ between 5 months and 7 years of age were selected for the trial at Barani Livestock Production Research Institute (BLPRI) Kherimurat, Attock. All the animals were sero-negative for PPR and there was no history of vaccination against PPR in selected animals. The duration of the experiment was 38 months. Animals were randomly allocated to three groups A, B and C. Each group comprised of 35 animals ( sheep $=15$ and goats $=20$ ). The animals in group A and B were administered subcutaneously in the sternum region with $1 \mathrm{ml}$ (recommended dose) and $0.5 \mathrm{ml}$ of an attenuated PPR virus vaccine Pestevac respectively (each dose contained PPR virus strain $75 / 1$ at a concentration of $10^{2.5}$ TCID $_{50}$ ) produced by Jordan Bio-In-dustries Centre (JOVAC). The animals in group C served as unvaccinated in contact control.

\subsection{Sample Collection and Analysis}

The sera of experimental animals were collected at monthly intervals during first year, at 2 months interval during second year and at 3 months interval during third year of the experiment. The sera were stored at $-20^{\circ} \mathrm{C}$ until tested. The samples were analyzed using anti-hemagglutinin $(\mathrm{H})$ monoclonal antibody based competitive ELISA (cELISA) [6] [7]. The cELISA kit was manufactured jointly by Biological Diagnostic supplies Limited (BDSL) with the collaboration of Flow Laboratories and Institute for Animal Health, Pirbright, Surrey, United Kingdom. The standardized reagents, bench protocol and manual were supplied along with the kit.

\subsection{Challenge Protection Studies}

The challenge protection studies were also carried out in the experimental animals. For this purpose three of the vaccinates (goats) which were immunised with normal recommended dose $(1 \mathrm{ml})$ of PPR vaccine and two unvaccinated in contact control goats were procured from BLPRI, Kherimurat. These animals were challenged subcutaneously in the neck region with $3 \mathrm{ml}$ of virus suspension (PAK-KP1-06/NARC3) at a concentration of $10^{3} \mathrm{TCID}_{50} / \mathrm{ml}$ at Animal Sciences Institute, National Agricultural Research Centre. The challenged animals were examined twice a day to monitor the development of clinical signs and the observations were recorded. The necropsy examination was carried out immediately after the death of each goat and the gross pathological lesions were recorded. The ocular, nasal and oral swabs were collected in $500 \mu \mathrm{l}$ of sterile phosphate buffer saline (PBS) from the challenged animals from day 0 to day 9 post challenge to find the evidence of viral shedding in challenged animals.

RNA was extracted from swab samples using Trizol reagent (Gibco BRL) following the manufacturer's instructions. One step PCR was performed in a 9902 thermal cycler (Applied Biosystems, Courtaboeuf, France). The extracted RNA was amplified using PPR virus specific primers based on highly conserved sequences within F gene of PPR virus [8]. Each PCR reaction contained $5 \mu 1$ of RNA, One step 5X RT-PCR Buffer (QIAGEN) with $12.5 \mathrm{mM} \mathrm{MgCl}_{2}, 10 \mathrm{mM}$ of each dNTP mix, $2 \mu$ enzyme mix, 5 units of RNAse inhibitor and 100 pmol of each of forward and reverse primers. The PCR conditions were as follows: an initial reverse transcription for 30 minutes at $50^{\circ} \mathrm{C}$, a PCR activation for 5 minutes at $95^{\circ} \mathrm{C}, 30$ cycles of amplification $\left(1\right.$ minute at $95^{\circ} \mathrm{C}, 1$ minute 
at $50^{\circ} \mathrm{C}$, and 2 minute at $72^{\circ} \mathrm{C}$ ), and a final extension step at $72^{\circ} \mathrm{C}$ for 10 minutes. The amplified PCR products were electrophoresed through $1.5 \%$ agarose gel in $1 \mathrm{X}$ TBE buffer at $90 \mathrm{~V}$ for 80 minutes and visualized using ultra violet illumination. The expected product size was $371 \mathrm{bp}$.

\subsection{Data Analysis}

The experimental animals were divided into three age categories: kids (5 months to 1 year old), young stock (1-2 years old) and adults (more than 2 years of age). One way analysis of variance (ANOVA) was used to assess the association of species, sex, age, treatment (vaccination against PPR) and time/month of sampling. This association was confirmed by repeated measures ANOVA. The multiple regression analysis that allowed for the random effects was used to validate the results of ANOVA. Intercooled STATA 9.2 was used for the statistical analysis.

\section{Results}

The animals with antibody levels sufficient to cause color inhibition (PI = percent inhibition) values more than $50 \%$ were considered positive and responsive to vaccination. The pre vaccination PI values (day 0 ) of all animals in the three groups were found to be diagnostically negative (PI values below 50\%) and ranged between 5 to $46 \%$. The highest mean PI value of more than $90 \%$ was observed on day 30 (one month) post vaccination in the animals in group A which received normal recommended dose of PPR vaccine. Thereafter, a steady decline was observed in the subsequent samplings at defined intervals. Significant antibody titres were observed even at the end of observation period i.e. 36 months post vaccination. A similar trend was observed in the animal vaccinated with half dose of PPR vaccine (group B). The highest antibody titre was observed 30 days post vaccination followed by a gradual decline in mean PI values and significant antibody titres were observed even at the end of observation period. The unvaccinated control animals (group C) did not develop any significant serological titres against PPR and remained negative throughout the observation period (Figure 1). The analysis also indicated weak association $(\mathrm{F}=4.58, \mathrm{P}=0.033)$ between sex and PI values of PPR cELISA. However, this may be attributed to the fact that only a small number of male animals $(n=5)$ were included in each group.

This study could not find an association between species and cELISA PI values (one way ANOVA $\mathrm{F}=0.02$, $\mathrm{P}=0.877$ ) and thus protection offered by PPR vaccine. The age of the animals was strongly associated with PI values ( $\mathrm{F}=28.44, \mathrm{P}=<0.001$ ). The Scheffe's multiple comparison test showed that the mean PI values among adults were significantly different from those of either young stock and kids (Table 1). These results suggest that immune response against PPR virus vaccination in adults is stronger than young stock and kids.

For univariate analysis, linear regression was used. There was no evidence of any association between the cELISA PI values and species of animals $(P=0.509)$ and sex $(P=0.394)$. Age of the animals appears to affect the PI values of the PPR cELISA $(\mathrm{P}=0.008)$ in the univariate analysis. However, the treatment group that an animal belonged to $(\mathrm{z}=22.08, \mathrm{P}<0.001)$ or the month of sampling $(\mathrm{z}=-8.00, \mathrm{P}<0.001)$ appeared to be strongly associated with the cELISA PI values. The final model contained the treatment group, month of sam-

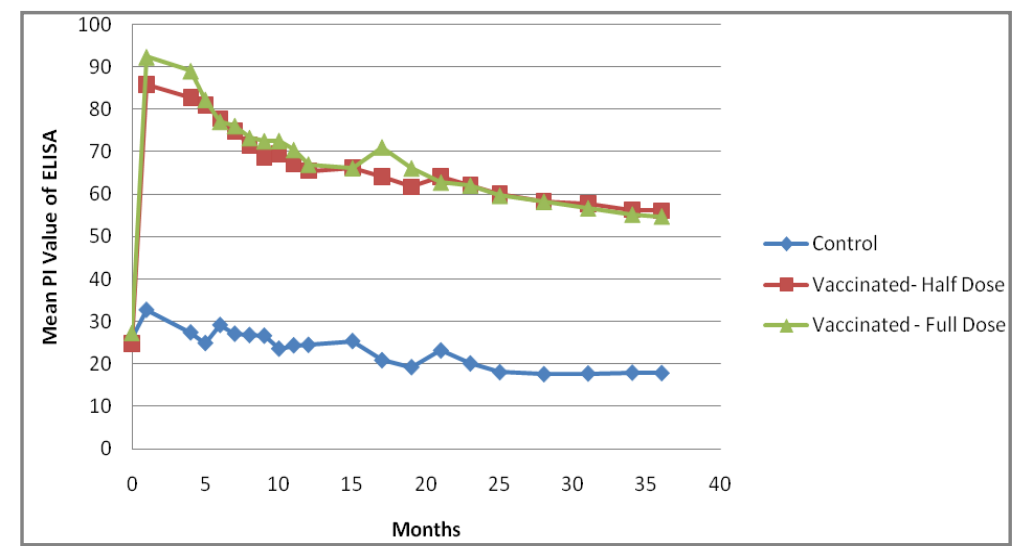

Figure 1. Mean percent inhibition (PI) values of PPR c-ELISA in animals among three treatment groups during 36 months of experiment. 
Table 1. Comparison of ELISA PI values by age groups (Scheffe’s multiple comparison test for means).

\begin{tabular}{ccc}
\hline \multirow{2}{*}{ Treatment } & \multicolumn{2}{c}{ Mean PI values } \\
\cline { 2 - 3 } & Kids & Young stock \\
\hline \multirow{2}{*}{ Young stock } & 3.92461 & \\
& $\mathrm{P}=0.06$ & 9.19332 \\
Adult & 13.1179 & $\mathrm{P}<0.001$ \\
\hline
\end{tabular}

pling and the age of animal at the start of trial. Multiple regression analysis for panel/longitudinal data was used for the final statistical analysis. Generalized least square (GLS) random effects estimation was used to allow for the mean titre to vary among various groups. After controlling for the effect of treatment group and month of sampling, the age appeared no longer associated with the PI values of PPR cELISA ( $\mathrm{z}=0.79, \mathrm{P}=0.43)$. The $\beta_{0}$ coefficient of regression was 28.46 (95\% C.I., $23.599-33.327: \mathrm{z}=11.47, \mathrm{P}<0.001)$. The treatment is quite strongly associated with the PI value. The beta coefficient of regression for treatment group $\left(\beta_{1}\right)$ was 37.972 (95\% C.I., $31.191-44.752, \mathrm{z}=11.70, \mathrm{P}<0.001)$. The beta coefficient of regression for month of sampling $\left(\beta_{2}\right)$ was -0.339 ( $95 \%$ C.I., -0.415 to $-0.263, \mathrm{z}=-8.73, \mathrm{P}<0.001$ ): there is a negative relation, i.e. the PI values decrease as the month of sampling gets farther from the date of vaccination. Assuming a linear relationship and after controlling for the effect of treatment group, the PI values can be determined as:

$$
P I \text { value }=28.463-0.339 \times \text { month of sampling }
$$

Similarly, the treatment group appeared to strongly affect the cELISA PI values. After controlling for the effect of month of sampling, the PI values were strongly associated with the treatment group.

Prediction:

The final regression equation takes the following form:

For the control group

$$
P I \text { value }=28.463+(0)-(0.339 \times \text { month of sampling })
$$

For the half dose vaccine group, it takes the following form;

$$
P I \text { value }=28.463+(37.972 \times 1)-(0.339 \times \text { month of sampling })
$$

Whereas for the full dose vaccine Group, it takes the following form:

$$
P I \text { value }=28.463+(43.492 \times 1)-(0.339 \times \text { month of sampling })
$$

Statistically, the PI values among both treatment groups are not different.

This statistical model was very good at fitting the observed PI values by controlling the variations among three treatment groups $\left(\mathrm{R}^{2}\right.$ between groups $\left.=0.849\right)$ and quite good at fitting the overall observed PI values $\left(\mathrm{R}^{2}\right.$ overall $=0.684$ ).

All the three challenged vaccinates withstood challenge and were found to be completely resistant clinically and virologically to virulent PPR virus. There was no evidence of virus shedding in ocular, oral and nasal secretions of three vaccinated animals examined using RT-PCR during the observation period (day 0 - day 9 post challenge). These animals remained protected and free of any clinical signs of PPR throughout the observation period i.e. 35 days post challenge.

The PPRV specific RNA was detected in the secretions (ocular, nasal and oral secretions) of the unvaccinated control animals on day 4 post challenge. Both the animals continued to shed virus till the end of observation period (day 9 post challenge).

The unvaccinated control animals exhibited fever (up to $41.2^{\circ} \mathrm{C}$ ) after an incubation period of four days accompanied by depression and anorexia. This was followed by ocular and nasal discharges, mouth lesions, diarrhea, and pneumonia culminating in death of animals on day 16 and day 21 post challenge. At necropsy the carcasses were dehydrated with sunken eyes and soiling of hind quarter with diarrhea material. The nasal and buccal mucosae were congested with red raw lesions on the cheek papillae in one of the animal. Both the lungs were 
found pneumonic. Hemorrhages were seen on the mucosal surface of the abomasum. There was hemorrhagic enteritis in large intestine (cecum and colon). The lymph-nodes particularly the mesenteric lymph nodes were found swollen along with accumulation of fluid in both the animals.

Second challenge protection study was conducted 36 months post vaccination. All the 4 challenged vaccinates withstood challenge and were completely found resistant clinically and virologically to virulent PPRV. The shedding of PPR virus was not observed in the ocular, nasal and oral swabs during the observation period. These animals remained protected and free of any clinical signs of PPR throughout the observation period i.e. 35 days post challenge.

The PPRV specific RNA was detected in the secretions (ocular, nasal and oral secretions) of the unvaccinated control animals on days 4 and 5 post challenge. Both the animals continued to shed virus till the end of observation period (day 9 post challenge). One of the unvaccinated control animal developed fever after an incubation period of four days. The second animal developed fever on day 5 post challenge. This was followed by ocular and nasal discharges; mouth lesions, diarrhea, and pneumonia followed by death of one animal on $23^{\text {rd }}$ day post challenge while the second goat recovered after a disease episode and remained alive till the end of observation period.

\section{Discussion}

Until recently the TCRV was used successfully for the induction of cross protection against PPR owing to the antigenic similarities between the two viruses [9]. It was demonstrated that TCRV was safe, efficacious and conferred protection in goats for 1 year [10] [11]. However, the use of TCRV was discontinued owing to the need for carrying out sero-epidemiological studies for the verification of presence of antibodies against rinderpest virus in ongoing rinderpest eradication campaign. A homologous PPR vaccine developed by successful attenuation of Nigeria 75/1 strain of PPRV on Vero cells became available in 1989 [12]. Unfortunately limited reports are available on the duration of immunity and protection conferred by this vaccine. Awa et al. [13] demonstrated that the titres remained above the protection threshold 12 months after the vaccination with PPR vaccine.

Although the experimental animals were from the area which is known to harbour PPRV, the pre-immunization sera antibody titres of all the experimental animals were found diagnostically negative for the presence of PPRV antibodies. A probable explanation is that the animals used in this study were stationed at isolated premises in an organized public sector farm with vast grazing areas. Under such conditions the chances of transmission of PPRV diminished when these animals had no contact with other animals of the area. Furthermore, there was no history of PPR at this farm. It was found that the vaccine virus was not transmitted to the in contact unvaccinated control animals. This observation is in agreement with those reported earlier [12] [14].

The highest serum antibody titres were recorded at 30 days post vaccination in goats and sheep vaccinated either with normal recommended dose or half dose of PPR vaccine. The antibody titres gradually increased from day to day and highest mean cELISA PI value of $81.5 \%$ were obtained at 30 days post vaccination in goats [15]. Maximum serum neutralization test tires were detected 1 month post vaccination in goats following vaccination with PPR vaccine [13]. This was followed by a gradual decrease in the mean PI values of the vaccinated animals during the entire study period (3 years post vaccination). The mean antibody titres remained diagnostically positive and above the protection threshold (more than 50\% cELISA PI values) in the last sampling 36 months post vaccination both in animals either vaccinated with recommended dose or half dose of PPR vaccine. It was found that even a reduced dose (half of the recommended dose) of PPR vaccine is capable of eliciting a strong immune response in the vaccinated animals. These findings are supported by the observations made during field trials with PPR vaccine where it was reported that the antibodies produced lasted for 3 years in respondent animals [9] [12]. The antibody levels declined between 12 and 18 months but then remained stable for 3 years or more after vaccination with TCRV in cattle [16].

A serological response with frequency distribution of PI values ranging between $50 \%-90 \%$ was observed in sheep and goats in response to PPR vaccination. These results are in agreement with those of Taylor [1] who reported that although sheep are less severely affected by PPRV than goats, yet a similar serological profile was exhibited by both species. It has been demonstrated that the entire small ruminant flocks sero-converted at the same time during an outbreak in Northern Cameroon [17]. However, a different sero-conversion rate following vaccination with PPR vaccine has been reported in sheep than goats where maximum titres were detected 4 
months post vaccination in sheep [13]. Likewise a different peak frequency distribution of PI values was reported for goats and sheep in convalescent sera tested positive for PPRV antibodies [18]. This variation may perhaps be due to the differences in breed, age, health status of animals and management conditions for the rearing of small ruminants. The outcome of PPR virus infection and its epidemiology may be affected by the breed. For example, the Guinean goat breeds are known to be highly susceptible to PPR [19].

A strong association existed between age of animals and antibody response to vaccination. The PI values of adult animals were significantly higher than those of young stock and kids. PPRV is known to cause severe disease with invariably fatal results in lambs, kids and in animals up to 2 years of age [11] [20]. This phenomenon of enhanced antibodies production in adult animals may be attributed to the increased immuno-competence and ability to launch strong humoral response on exposure to PPRV. These results are in accordance with Abubakar et al. [21] who reported a higher prevalence of antibodies against PPRV in adult sheep and goats (more than 2 years of age). Also the proportion of sero-positive animals increases with age for PPR [22].

There was no evidence of virus shedding in the ocular, nasal and oral secretions of vaccinated animals during the challenge protection studies. All the vaccinated animals challenged after 24 and 36 months post vaccination remained protected. In Morbillivirus infection, association between the neutralizing antibody titres and resistance to natural or experimental challenge following vaccination is often emphasized [16]. It is evident by the kinetics of antibody response in challenged animals that a strong anamnestic (secondary) response was not observed in challenged animals 24 months post vaccination. Similar retarded serological responses have been reported in cattle challenged with virulent rinderpest virus 6 to 11 years after vaccination with TCRV [16]. However, a significant rise in antibodies titres was observed in the animals challenged 36 months post vaccination. It was found that animals with weakly positive antibody titres and even with diagnostically negative titres resisted challenge and remained protected 36 months post vaccination. Plowright [16] demonstrated that vaccinates which were negative or weakly positive for antibodies against rinderpest virus resisted challenge with virulent rinderpest virus.

Though sheep and goats vaccinated with half dose of PPR vaccine were not challenged, yet looking at humoral response in these animals it can be speculated that they might have withstood challenge and remained protected. These results however should not be considered conclusive because only a small number of animals were used for challenge owing to financial constraints and insufficient facilities needed for the management of challenged animals. However, these results are strongly suggestive of the protection conferred by a single subcutaneous injection of PPR vaccine 3 years after vaccination.

The role of cell mediated immunity in the protection against challenge with virulent PPRV has not been investigated. It is difficult to distinguish the relative importance of the role of cell mediated and humoral immunity in either recovery from infection or protection against challenge by vaccination [23]. The fusion protein of PPRV has been reported to play an important role in cell mediated immunity [9]. Further studies in this regard may prove helpful in understanding the phenomena of protection in natural PPRV infection and in experimental challenge.

\section{Conclusion}

The economic life of small ruminants is about 3 years. This study indicated that PPR vaccine (Nigerian strain 75/1) provides immunity for 3 years. Therefore, the strategic use of this vaccine will be useful in reducing the impact of infection in the areas where the disease is endemic.

\section{Acknowledgements}

Assistance of FAO projects GCP/PAK/127/USA-Progressive control of Peste des Petits Ruminants in Pakistan and GTFS/INT/907/ITA-Controlling Transboundary Animal Diseases in Central Asian Countries in undertaking this study is highly acknowledged.

\section{References}

[1] Gibbs, E., Taylor, W., Lawman, M. and Bryant, J. (1979) Classification of Peste des Petits Ruminants Virus as the Fourth Member of the Genus Morbillivirus. Intervirology, 11, 268-274. http://dx.doi.org/10.1159/000149044

[2] Baldock, C., Forman, T., Geering, B. and Taylor, B. (1999) New Technologies in the Fight Against Transboundary 
Animal Diseases. Rome.

[3] Amjad, H., Forsyth, M., Barrett, T. and Rossiter, P. (1996) Peste des Petits Ruminants in Goats in Pakistan. Veterinary Record, 139, 118-119. http://dx.doi.org/10.1136/vr.139.5.118

[4] Zahur, A., Irshad, H., Hussain, M., Ullah, A., Jahangir, M., Khan, M.Q. and Farooq, M.S. (2008) The Epidemiology of Peste des Petits Ruminants in Pakistan. Rev Sci Tech (International Office of Epizootics), 27, 877-884.

[5] Zahur, A., Ullah, A., Hussain, M., Irshad, H., Hameed, A., Jahangir, M. and Farooq, M. (2011) Sero-Epidemiology of Peste des Petits Ruminants (PPR) in Pakistan. Preventive Veterinary Medicine, 102, 87-92.

http://dx.doi.org/10.1016/j.prevetmed.2011.06.011

[6] Anderson, J. and McKay, J.A. (1994) The Detection of Antibodies Against Peste des Petits Ruminants Virus in Cattle, Sheep and Goats and the Possible Implications to Rinderpest Control Programmes. Epidemiology \& Infection, 112, 225-231. http://dx.doi.org/10.1017/S0950268800057599

[7] Libeau, G., Prehaud, C., Lancelot, R., Colas, F., Guerre, L., Bishop, D. and Diallo, A. (1995) Development of a Competitive ELISA for Detecting Antibodies to the Peste des Petits Ruminants Virus Using a Recombinant Nucleoprotein. Research in Veterinary Science, 58, 50-55. http://dx.doi.org/10.1016/0034-5288(95)90088-8

[8] Forsyth, M. and Barrett, T. (1995) Evaluation of Polymerase Chain Reaction for the Detection and Characterization of Rinderpest and Peste de Petit Ruminant's Viruses for Epidemiological Studies. Virus Research, 39, 151-163. http://dx.doi.org/10.1016/0168-1702(95)00076-3

[9] Diallo, A., Minet, C., Le Goff C., Berhe, G., Albina, E., Libeau, G. and Barrett T. (2007) The Threat of Peste des Petits Ruminants: Progress in Vaccine Development for Disease Control. Vaccine, 25, 5591-5597. http://dx.doi.org/10.1016/j.vaccine.2007.02.013

[10] Adu, F. and Nawathe D. (1981) Safety of Tissue Culture Rinderpest Vaccine in Pregnant goats. Tropical Animal Health and Production, 13, 166-166. http://dx.doi.org/10.1007/BF02237916

[11] Taylor, W. (1984) The Distribution and Epidemiology of Peste des Petits Ruminants. Preventive Veterinary Medicine, 2, 157-166. http://dx.doi.org/10.1016/0167-5877(84)90059-X

[12] Diallo, A., Taylor, W.P., Lefevre, P.C. and Provost, A. (1989) Attenuation D'une Ouche De Virus De La Peste des Petits Ruminants: Candidat Pour un Vaccin Homologue Vivant. Rev Elev Med Vet Pay, 42, 311-319.

[13] Awa, DN., Ngagnou, A., Tefiang, E., Yaya, D, and Njoya, A. (2002) Post Vaccination and Colostral Peste des Petits Ruminants Antibody Dynamics in Research Flocks of Kirdi Goats and Foulbe Sheep of North Cameroon. Preventive Veterinary Medicine, 55, 265-271. http://dx.doi.org/10.1016/S0167-5877(02)00013-2

[14] Intizar, M., Ahmad, M., Anjum, A, and Hanif, A. (2009) Comparative Efficacy of Peste des Petits Ruminants (PPR) Vaccines Available in Pakistan in Sheep and Goats. Pakistan Veterinary Journal, 29.

[15] Siddique, M., Rahman, M., Chowdhury, S., Kafi, M. and Alam, M. (2006) Determination of Efficacy of Thermostable PPR Live Homologous Vaccine Incubated at Room Temperature for 14 Days. BangladeshJVetMed, 4, 43-46.

[16] Plowright, W. (1984) The Duration of Immunity in Cattle following Inoculation of Rinderpest Cell Culture Vaccine. Journal of Hygiene-Cambridge, 92, 285-296. http://dx.doi.org/10.1017/S0022172400064524

[17] Martrenchar, A., Bouchel, D., Zoyem, N., Thiaucourt, F. and Lambert, M. (1997) Risk Factors Responsible for the Appearance of Individual Clinical Signs in Small Ruminants in Northern Cameroon. Small Ruminant Research, 26, 45-52. http://dx.doi.org/10.1016/S0921-4488(96)00975-3

[18] Singh, R., Saravanan, P., Sreenivasa, B., Singh, R. and Bandyopadhyay, S. (2004) Prevalence and Distribution of Peste des Petits Ruminants Virus Infection in Small Ruminants in India. Rev Sci Tech (International Office of Epizootics), 23, 807-819.

[19] Lefevre, P.C, and Diallo, A. (1990) Peste des Petits Ruminants. Rev Sci Tech (International Office of Epizootics), 9, 935-981.

[20] Roeder, P.L. and Obi, T.U. (1999) Recognizing Peste des Petits Ruminants: A Field Manual. In: Food and Agriculture Organization of the United Nations. Rome. ftp://ftp.fao.org/docrep/FAO/003/X1703E/X1703E00.PDF.

[21] Abubakar, M., Ali, Q. and Khan, H.A. (2008)Prevalence and Mortality Rate of Peste des Petitis Ruminant (PPR): Possible Association with Abortion in Goat. Tropical Animal Health and Production, 40, 317-321. http://dx.doi.org/10.1007/s11250-007-9105-2

[22] Losos, G.J. (1986) Infectious Tropical Diseases of Domestic Animals. Longman Scientific \& Technical.

[23] Cosby, S.L., Kai, C. and Yamanouchi, K. (2005) Immunology of Rinderpest-An Immunosuppres-sion but a Lifelong Vaccine Protection. In: Barrett, T., Paul-Pierre, P. and Taylor, W., Eds. Rinderpest and Peste des Petits Ruminants: Virus Plagues of Large and Small Ruminants. Pirbright, United Kingdom, 196. 